\title{
One Property of Bregman Projection onto Closed Convex Sets in Banach Spaces and Applications
}

\author{
Yong Zhang \\ School of Mathematics, Chengdu University of Information Technology \\ Chengdu 610225, China \\ E-mail: jkxzhangyong@cuit.edu.cn \\ Quangang Li \\ School of Mathematics, Chengdu University of Information Technology \\ Chengdu 610225, China \\ E-mail: quangang.1@163.com \\ Xiyao Zou \\ School of Mathematics, Chengdu University of Information Technology \\ Chengdu 610225, China \\ E-mail: zouxiyao110@163.com
}

Received: April 8, 2011 Accepted: May 12, 2011 Published: November 1, 2011

doi:10.5539/jmr.v3n4p55 URL: http://dx.doi.org/10.5539/jmr.v3n4p55

\begin{abstract}
We study one property of Bregman projection onto closed convex sets in Banach spaces. As an application, we use this property to simplify a class of hybrid algorithms for finding fixed points of nonlinear mappings and solving convex feasibility, variational inequality and equilibrium problems. This will reduce the computational complexity.
\end{abstract}

Keywords: Fixed points, Bregman projection, Hybrid method, Generalized projection, Iterative algorithm

\section{Introduction}

In this paper, we assume that $E$ is a real Banach space with norm $\|\cdot\|$ and let $E^{*}$ be the dual of $E$. We denote by $J$ the normalized duality mapping from $E$ to $2^{E^{*}}$ defined by

$$
J(x)=\left\{f \in E^{*}:\langle x, f\rangle=\|f\|^{2}=\|x\|^{2}\right\},
$$

where $\langle\cdot, \cdot\rangle$ denotes the generalized duality pairing.

Let $f: E \rightarrow(-\infty,+\infty]$ be a proper convex function with its domain $\operatorname{dom} f$. For any $x \in \operatorname{dom} f$ and $z \in E$ we denote by $f_{+}^{\prime}(x, z)$ the right-hand side derivative off at $x$ in the direction $z$, that is

$$
f_{+}^{\prime}(x, z):=\lim _{t \rightarrow 0^{+}} \frac{f(x+t z)-f(x)}{t} .
$$

The Bregman distance with respect to $f$ is defined by the function $D_{f}: \operatorname{dom} f \times \operatorname{dom} f \rightarrow[0,+\infty]$

$$
D_{f}(y, x):=f(y)-f(x)-f_{+}^{\prime}(x, y-x) .
$$

The function $D_{f}$ is not a distance in the usual sense: it is not always symmetric and may not satisfy the triangular inequality. For the more details and other related results, the readers are referred to (L.M. Bregman, 1967, p200-217), (D. Butnariu, E. Resmerita, 2006, p1-39), (C. Li, W. Song, J.C. Yao, 2010, p1128-1149).

Denote $P$ be the metric projection. As we know, if $C$ is a nonempty closed convex subset of a real Hilbert space then the metric projection $P_{C}: H \rightarrow C$ is nonexpansive. It is not available in more general Banach spaces. In this connection, Alber (Ya.I. Alber, 1996, p15-50) introduced generalized projection, denoted by $\Pi$, which is analogous to the metric projection $P$ in a Hilbert space. 
Similarly, by using the Bregman distance, Lve Bregman (L.M. Bregman, 1967, p200-217) introduced Bregman projection with respect to $f$, denoted by $\Pi^{f}$, which is natural generalization of the generalized projection $\Pi$. This presented an elegant and effective technique in designing and analyzing iterative algorithms for solving feasibility and optimization problems, variational inequalities, equilibrium problems and finding fixed points of nonlinear mappings.

In this paper, we firstly show a fact that if $C$ is a nonempty closed convex subset and $x_{0}$ is not in the interior of $C$, then the Bregman projection from $x_{0}$ on $C$ must be a subset of the boundary of $C$. As an application, we use this property to simplify a class of hybrid algorithms which were studied in (S. Reich, S. Sabach, 2010, p122-135). This will reduce the computational complexity. The simpler form algorithms also have the convergence theorems.

\section{Preliminaries}

Let $C \subset \operatorname{dom} f$ be a nonempty subset. For any $x_{0} \in \operatorname{dom} f$, the Bregman projection on $C$ with respect to $f$ is defined as the set of solutions of the optimization problem $\min _{y \in C} D_{f}\left(y, x_{0}\right)$, that is,

$$
\Pi_{C}^{f}\left(x_{0}\right):=\arg \min _{y \in C} D_{f}\left(y, x_{0}\right) .
$$

It is well known that if $E$ is smooth, then the normalized duality mapping $J$ is single valued. In this case, we consider the Liapunov functional $\phi: E \times E \rightarrow[0,+\infty]$ defined by

$$
\phi(y, x)=\|y\|^{2}-2\langle J(x), y\rangle+\|x\|^{2} \quad \forall x, y \in E .
$$

Let $K$ be a nonempty subset of $E$. The generalized projection operator $\Pi_{K}: E \rightarrow K$ is a map that assigns to an arbitrary point $x_{0}$ in $E$ the minimal points set of the functional $\phi(x, y)$, that is

$$
\Pi_{K}\left(x_{0}\right)=\left\{z \in K: \phi\left(z, x_{0}\right)=\min _{y \in K} \phi\left(y, x_{0}\right)\right\} .
$$

The function $f$ is called Gâteaux differentiable at $x$ if $\lim _{t \rightarrow 0}(f(x+t z)-f(x)) / t$ exists for any $z$. In this case $f_{+}^{\prime}(x, z)$ coincides with $\nabla f(x)$, the value of the gradient $\nabla f$ of $f$ at $x$. Thus, $D_{f}(y, x)=f(y)-f(x)-\langle\nabla f(x), y-x\rangle$. The function $f$ is said to be Fréchet differentiable at $x$ if this limit is attained uniformly in $y$ with $\|y\|=1$.

It is observed that in a smooth Banach space if $f(x)=\|x\|^{2}$ then the functional $D_{f}(y, x)$ coincides with $\phi(y, x)$ and $\Pi_{C}^{f}=\Pi_{C}$. While if $E$ is a real Hilbert space and $f(x)=\|x\|^{2}$ then $\Pi_{C}^{f}=\Pi_{C}=P_{C}$. In particular, if $x_{0} \in C$ then $\Pi_{C}^{f}\left(x_{0}\right)=x_{0}, \Pi_{C}\left(x_{0}\right)=x_{0}$ and $P_{C}\left(x_{0}\right)=x_{0}$.

Recall that a subset of a topological space is closed if and only if it contains its boundary. A subset of a topological space has empty boundary if and only if it is both open and closed. Let $U$ be a nonempty closed subset of a topological space, we use int $U$ to denote the interior of $U$ and $\mathrm{b}(U)$ to denote the boundary of $U$.

\section{Main results}

Theorem 3.1 Let $f$ be a proper convex function and $C \subset \operatorname{dom} f$ be a nonempty closed convex subset with nonempty boundary. For any fixed $x_{0} \in \operatorname{dom} f$ and $x_{0} \notin \operatorname{int} C$, if the minimum value of $D_{f}(y, x)$ exists on $C$, then it must be attained on $\mathrm{b}(C)$, i.e., $\Pi_{C}^{f}\left(x_{0}\right) \subset \mathrm{b}(C)$.

Proof. If int $C=\emptyset$, then it is easy. Since $C$ is a nonempty closed convex subset, it implies that $C=\mathrm{b}(C)$. It is obvious that $\Pi_{C}^{f}\left(x_{0}\right) \subset C=\mathrm{b}(C)$.

Now, we assume that int $C \neq \emptyset$. Suppose on the contrary that $D_{f}(y, x)$ can not obtain its minimum value on $\mathrm{b}(C)$. Hence, there exists a point $z \in \operatorname{int} C$ such that

$$
D_{f}\left(z, x_{0}\right) \leq D_{f}\left(y, x_{0}\right) \quad \forall y \in \mathrm{b}(C) .
$$

Because $x_{0} \notin \operatorname{int} C$ and $C$ is a nonempty closed convex subset, there exists a point $y_{0}=(1-t) x_{0}+t z, t \in[0,1)$, such that $y_{0} \in \mathrm{b}(C)$. Since $f$ is convex, the function $D_{f}(\cdot, x)$ is also convex. Thus, for any $t \in[0,1)$, we have

$$
0 \leq D_{f}\left(z, x_{0}\right) \leq D_{f}\left(y_{0}, x_{0}\right) \leq(1-t) D_{f}\left(x_{0}, x_{0}\right)+t D_{f}\left(z, x_{0}\right)=t D_{f}\left(z, x_{0}\right) .
$$

This is a contradiction. Therefore $\Pi_{C}^{f}\left(x_{0}\right) \subset \mathrm{b}(C)$. The proof is complete.

If we take $f(x)=\|x\|^{2}$ then $\Pi_{C}^{f}=\Pi_{C}$. So, we can obtain the following result form Theorem 3.1.

Corollary 3.1 Let $C$ be a nonempty closed convex subset of smooth Banach space E and with nonempty boundary. For any fixed $x_{0} \in E$ and $x_{0} \notin \operatorname{int} C$, if the minimum value of $\phi(y, x)$ exists on $C$, then it must be attained on $\mathrm{b}(C)$, i.e., $\Pi_{C}\left(x_{0}\right) \subset \mathrm{b}(C)$. 


\section{Applications}

By using the Bregman projection $\Pi^{f}$, Reich and Sabach (S. Reich, S. Sabach, 2010, p122-135) studied two hybrid algorithms for finding common fixed points of finitely many Bregman strongly nonexpansive operators. They established strong convergence theorems and then applied them to the solution of convex feasibility, variational inequality and equilibrium problems. In this section, we use Theorem 3.1 to simplify those algorithms which were studied in (S. Reich, S. Sabach, 2010, p122-135) in order to reduce the computational complexity.

It does not matter to our result if those algorithms have computational errors or not. For the sake of simplicity, we remove the computational errors. Without loss of generality, we quote the following algorithm:

$$
\left\{\begin{array}{l}
y_{n}^{i}=T_{i}\left(x_{n}\right), x_{0} \in E, C_{0}^{i}=E, i=1,2, \cdots, N, \\
C_{n+1}^{i}=\left\{z \in C_{n}^{i}: D_{f}\left(z, y_{n}^{i}\right) \leq D_{f}\left(z, x_{n}\right)\right\}, \\
C_{n+1}:=\bigcap_{i=1}^{N} C_{n+1}^{i}, \\
x_{n+1}=\prod_{C_{n+1}}^{f}\left(x_{0}\right) \quad n \in \mathbb{N},
\end{array}\right.
$$

where $E$ is a reflexive Banach space, $T_{i}$ are Bregman strongly nonexpansive operators and $f: E \rightarrow \mathbb{R}$ is a Legendre function which is bounded, uniformly Fréchet differentiable and totally convex on a bounded subset of $E$.

Our idea is to replace the $C_{n+1}^{i}$ in (4.1) with a new closed convex sets which is smaller than $C_{n+1}^{i}$ but have the same boundary whit $C_{n+1}^{i}$. So the iteration processes will be simpler than the primary one in calculating $C_{n+1}, C_{n+2}^{i}$ and projection points.

We simplify (1) as follows:

$$
\left\{\begin{array}{l}
y_{n}^{i}=T_{i}\left(x_{n}\right), x_{0} \in E, C_{0}^{i}=E, i=1,2, \cdots, N, \\
\widehat{C_{n+1}^{i}}=\left\{z \in C_{n}^{i}: D_{f}\left(z, y_{n}^{i}\right)=D_{f}\left(z, x_{n}\right)\right\}, \\
\widehat{C_{n+1}}:=\bigcap_{i=1}^{N} \widehat{C_{n+1}^{i}}, \\
x_{n+1}=\Pi_{\widehat{C_{n+1}}}^{f}\left(x_{0}\right) \quad n \in \mathbb{N} .
\end{array}\right.
$$

Proposition 4.1 The schemes (1) and (2) must generate the same sequence $\left\{x_{n}\right\}$ hence they have the same convergence theorem.

Proof. As in the proof of (S. Reich, S. Sabach, 2010, p122-135), under the assumptions of $f$, they ensured the Bregman Projection onto closed convex sets have a unique element and also claimed that $C_{n+1}^{i}$ are closed and convex. Next, we prove that $\widehat{C_{n+1}^{i}}$ are also closed and convex sets and $\mathrm{b}\left(C_{n+1}\right)=\mathrm{b}\left(\widehat{C_{n+1}}\right)$.

As a matter of fact, the defined inequality of $C_{n+1}^{i}$ in (1) is equivalent to the inequality

$$
\left\langle\nabla f\left(x_{n}\right)-\nabla f\left(y_{n}^{i}\right), z\right\rangle \leq f\left(y_{n}^{i}\right)-f\left(x_{n}\right)+\left\langle\nabla f\left(x_{n}\right), x_{n}\right\rangle-\left\langle\nabla f\left(y_{n}^{i}\right), y_{n}^{i}\right\rangle .
$$

This inequality is affine in $z$. Similarly, the defined inequality of $\widehat{C_{n+1}^{i}}$ in (2) is equivalent to the inequality

$$
\left\langle\nabla f\left(x_{n}\right)-\nabla f\left(y_{n}^{i}\right), z\right\rangle=f\left(y_{n}^{i}\right)-f\left(x_{n}\right)+\left\langle\nabla f\left(x_{n}\right), x_{n}\right\rangle-\left\langle\nabla f\left(y_{n}^{i}\right), y_{n}^{i}\right\rangle .
$$

Since $C_{n+1}^{i}$ are closed and convex, it follows that $\widehat{C_{n+1}^{i}}$ also are closed and convex, and $\mathrm{b}\left(C_{n+1}^{i}\right)=\mathrm{b}\left(\widehat{C_{n+1}^{i}}\right)$. Hence, $\mathrm{b}\left(C_{n+1}\right)=\mathrm{b}\left(\widehat{C_{n+1}}\right)$.

Note that, after finite iterations, we must have $x_{0} \notin \operatorname{int} C_{n+1}$. If not, then $\left\{x_{n}\right\} \equiv\left\{x_{0}\right\}$. Only when $x_{0} \in \bigcap_{i=1}^{N} F\left(T_{i}\right)$, will this situation happen.

Since $x_{0} \notin \operatorname{int} C_{n+1}$, it follows from $\mathrm{b}\left(C_{n+1}\right)=\mathrm{b}\left(\widehat{C_{n+1}}\right)$ and Theorem 3.1 that $x_{n+1}=\Pi_{C_{n+1}}^{f}\left(x_{0}\right)=\Pi_{\widehat{C_{n+1}}}^{f}\left(x_{0}\right) \in \mathrm{b}\left(C_{n+1}\right)$. The proof is complete.

Remark 4.1 In general, in order to show $C_{n+1}^{i}$ are closed, only $f$ need to have some conditions to ensure the continuity of the right-hand side derivative of $f$. For example (S. Reich, S. Sabach, 2010, p122-135), if $f$ is uniformly Fréchet differentiable and bounded on a bounded subset $S$ of $E$, then $\nabla f$ is uniformly continuous on $S$ from the strong topology of $E$ to the strong topology of $E^{*}$. But if we want to establish convergence theorem, more assumptions may be needed.

Remark 4.2 Similarly to the Bregman projection in Banach spaces, metric projections and generalized projections also have this property. We can simplify many types of hybrid algorithms such as CQ method (K. Nakajo, W. Takahashi, 2003, 
p372-379), monotone hybrid algorithm (Y.F. Su, D.X. Wang, M.J. Shang, 2008, p1-8) and shrinking projection method (W. Takahashi, Y. Takeuchi, R. Kubota, 2008, p276-286) by using this idea to reduce the computational complexity.

\section{References}

C. Li, W. Song, J.C. Yao. (2010). The Bregman distance, approximate compactness and convexity of Chebyshev sets in Banach spaces. J. Approx. Theory, 162, 1128-1149.

D. Butnariu, E. Resmerita. (2006). Bregman distances, totally convex functions and a method for solving operator equations in Banach spaces. Abstr. Appl. Anal., Volume2006, 1-39. http://dx.doi.org/10.1155/AAA/2006/84919

K. Nakajo, W. Takahashi. (2003). Strong convergence theorems for nonexpansive mappings and nonexpansive semigroups. J. Math. Anal. Appl., 279, 372-379. http://dx.doi.org/10.1016/S0022-247X(02)00458-4

L.M. Bregman. (1967). The relaxation method for finding the common point of convex sets and its application to the solution of problems in convex programming. USSR Comput. Math. and Math. Phys., 7, 200-217.

S. Reich, S. Sabach. (2010). Two strong convergence theorems for Bregman strongly nonexpansive operators in reflexive Banach spaces. Nonlinear Anal., 73, 122-135. http://dx.doi.org/10.1016/j.na.2010.03.005

W. Takahashi, Y. Takeuchi, R. Kubota. (2008). Strong convergence theorems by hybrid methods for families of nonexpansive mappings in Hilbert spaces. J. Math. Anal. Appl., 341, 276-286. http://dx.doi.org/10.1016/j.jmaa.2007.09.062

Ya.I. Alber. (1996). Metric and generalized projection operators in Banach spaces: properties and Applications, in: A.G. Kartsatos (Ed.), Theory and Applications of Nonlinear Operators of Accretive and Monotone Type, New York:Marcel Dekker, (pp. 15-50).

Y.F. Su, D.X. Wang, M.J. Shang. (2008). Strong Convergence of Monotone Hybrid Algorithm for Hemi-Relatively Nonexpansive Mappings. Fixed Point Theory and Applications, Volume 2008, 1-8. http://dx.doi.org/10.1155/2008/284613 